\title{
Demagnetization Diagnosis of Permanent Magnet Synchronous Motor Using Frequency Analysis at Standstill Condition
}

\author{
Jin-Hyung Yoo and Tae-Uk Jung* \\ Department of Electrical Engineering, Kyungnam University, Changwon 51767, Korea
}

(Received 23 February 2016, Received in final form 18 May 2016, Accepted 23 May 2016)

\begin{abstract}
Recently, electric vehicles have got significant attention because it is more eco-friendly and efficient than internal combustion engine vehicles. Instead of an internal combustion engine, the electric vehicle has a motor for propulsion. The permanent magnet synchronous motor which has permanent magnet instead of field winding in the rotor has especially higher efficiency and power density than other types of motor. When the irreversible demagnetization is occurred, drivers are exposed to high risk of accident by the fault operation of motor. Therefore, the irreversible demagnetization of permanent magnet should be detected to reduce the risk of accident. In this study, the demagnetization diagnosis method based on the result of locked rotor test is proposed. Based on short measurement time, the proposed diagnosis method aims to detect the demagnetization fault when an electric vehicle is at a complete standstill. The proposed method is verified through the finite element analysis.
\end{abstract}

Keywords : Demagnetization, Frequency analysis, Permanent magnet, Electric Vehicle

\section{Introduction}

The permanent magnet synchronous motor (PMSM) which has permanent magnet instead of field winding in the rotor has especially higher efficiency and torque per weight than other types of motor. However, permanent magnet $(\mathrm{PM})$ can be irreversibly demagnetized by the temperature variation, permeance coefficient and external magnetic field. When the demagnetization is occurred, users can be exposed to high risk of accident by the fault operation of motor.

Therefore, the demagnetization of PM should be detected by using any kinds of methods. In general, the diagnosis of irreversible demagnetization is implemented using the gauss meter or stator current spectrum. The gauss meter directly measures the flux density of magnet accurately but a motor should be disassembled to measure the flux density. When the motor is operating, some detection methods for the demagnetization of PM have been presented [1-7]. However, each method has major drawbacks because it requires an additional sensor such as current sensor, raster encoder and search coils.

(C)The Korean Magnetics Society. All rights reserved.

*Corresponding author: Tel: +82-55-249-2628

Fax: +82-505-999-2161, e-mail: tujung@kyungnam.ac.kr
In this study, the demagnetization diagnosis method based on locked rotor test and frequency analysis is proposed. The proposed diagnosis method aims to regular diagnosis at standstill condition through frequency analysis of current which caused by the rotating magnetic field of stator winding.

The proposed method has an advantage for frequency analysis because the fundamental frequency of current is fixed by input voltage for diagnosis [8, 9]. However, PM can be demagnetized by the high current in stator winding. In order to prevent demagnetization through the proposed method, the low voltage high frequency signal is used. The high frequency of input voltage also helps to reduce torque production of PMSM. The proposed method is verified through the finite element analysis (FEA).

\section{Existing Demagnetization Diagnosis Methods}

\subsection{Causes of demagnetization fault}

Faults of permanent magnet synchronous motor can be classified as electrical faults, mechanical faults and magnetic faults. Demagnetization fault is a kind of magnetic fault of PMSM.

Permanent magnets can be demagnetized as the position of operating point moved to the negative side over the 


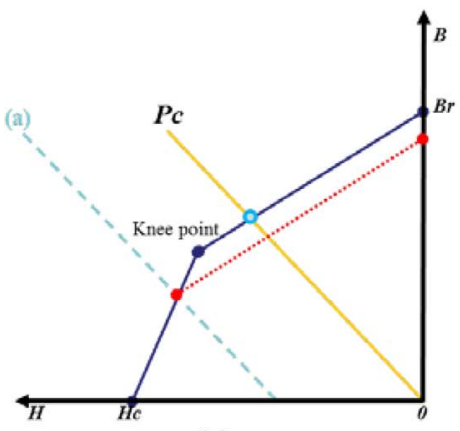

(a)

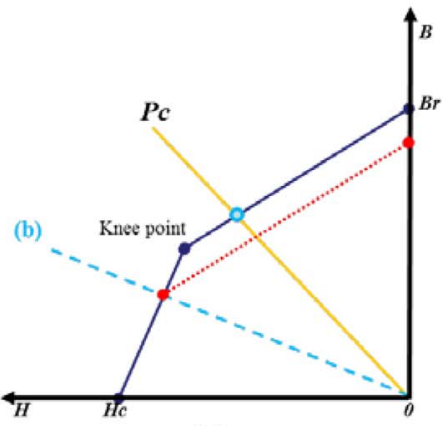

(b)

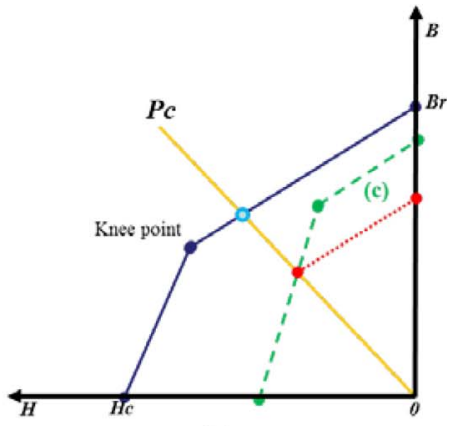

(c)

Fig. 1. (Color online) (a) External magnetic field, (b) Permeance coefficient, (c) Temperature variation.

knee point by the external magnetic field, permeance coefficient and temperature variation as shown in Fig. 1. Operating point of PM is the intersecting point of the B-H curve and permeance coefficient line.

For example, when the partial demagnetization is occurred, the stator current should be increased to generate the same output torque. As a result of increased current, inverse magnetic field that opposes the remnant induction of the permanent magnets are increased. In addition, the temperature of the PMSM is augmented by increased copper loss. Because of the increased factors, the demagnetization of permanent magnets is getting worse. Because of this, the demagnetization should be detected to prevent the fault operation of PMSM.

\subsection{Existing demagnetization diagnosis methods}

The simplest alternative for demagnetization diagnosis is to collect data from the terminal voltages at the regenerative braking condition as shown in Fig. 2.

However, rotor speed and back electromotive force

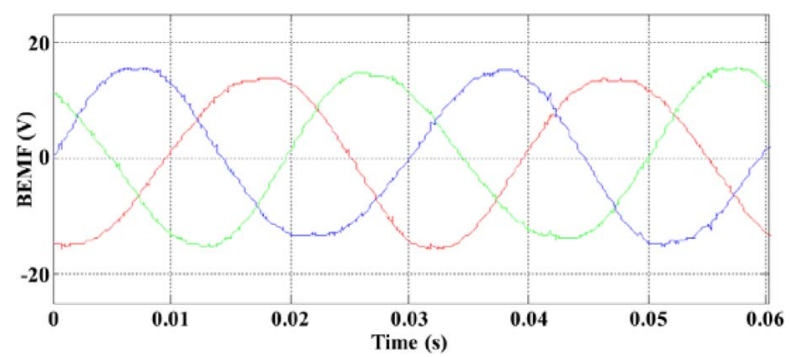

Fig. 2. (Color online) Measurement result of terminal voltage at the regenerative braking condition. constant should be measured very accurately. Furthermore, this method is improper to detect partial demagnetization of distributed winding type PMSM.

Another alternative is the stator current frequency analysis. When the motor is operating, this method detects demagnetization of PM. However, this method is not good when a motor speed is varying because the fundamental frequency of stator current is varied by motor speed.

The other types of methods require accurate parameters of fault-free machine or additional built-in sensors for diagnosis. The hardware requirement and drawbacks of existing diagnosis methods are summarized in Table 1.

\section{Proposed Demagnetization Diagnosis Method}

\subsection{Demagnetization modeling of target PMSM}

Figure 3 shows the 2D model and winding diagram of interior permanent magnet synchronous motor (IPMSM) used in this study. The winding type of target IPMSM is
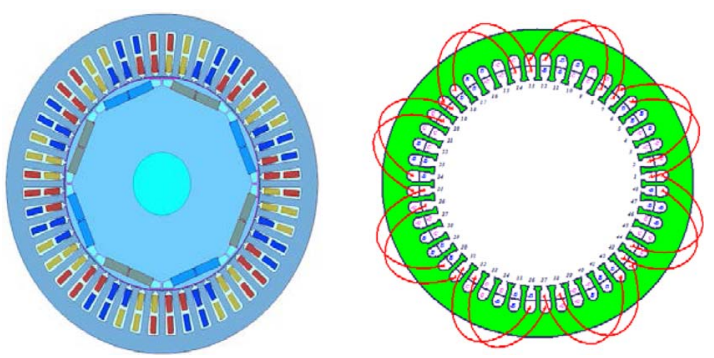

Fig. 3. (Color online) 2D FEA model and winding diagram of target PMSM.

Table 1. Comparison of diagnosis techniques for demagnetization.

\begin{tabular}{ccc}
\hline \hline Types of methods & Hardware requirement & Drawbacks \\
\hline Stator current frequency analysis & Current sensor, Raster encoder & Not good for various speed operated machines \\
Parameter estimation based on current and & Current sensor, Raster encoder & Accurate parameters of fault-free machine are required \\
voltage monitoring & Search coils, Raster encoder & Additional built-in flux sensors are required \\
Direct flux monitoring & . & A
\end{tabular}


Table 2. Specifications of target PMSM.

\begin{tabular}{cc}
\hline \hline Parameter & Value \\
\hline Rated output power $[\mathrm{kW}]$ & 30 \\
Number of slots & 48 \\
Number of poles & 8 \\
Number of turns in series & 20 \\
Number of parallel branches & 8 \\
Stack length [mm] & 140 \\
Core material & $\mathrm{S} 18$ \\
Permanent magnet & NdFeB 35EH \\
\hline
\end{tabular}

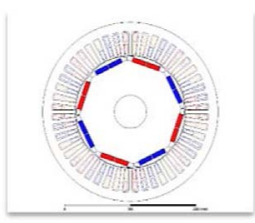

(a)

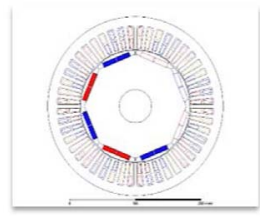

(d)

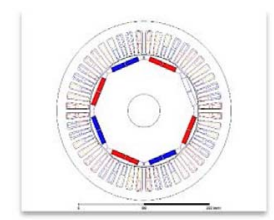

(b)

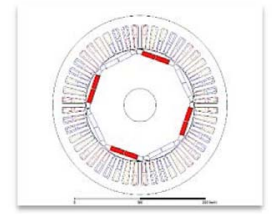

(e)

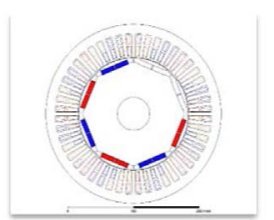

(c)

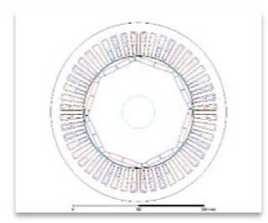

(f)
Fig. 4. (Color online) Demagnetization analysis model of target PMSM.

double layer distributed winding. Specifications of target IPMSM are summarized in Table 2.

Figure 4 shows the demagnetization analysis model of the target IPMSM. In Figure 4, transparent poles are demagnetized. The arrangement of demagnetized poles is determined arbitrarily. In this study, it is assumed that the demagnetized poles have $70 \%$ reduced coercive force and residual magnetic flux density.

\subsection{Demagnetization diagnosis by terminal voltage measurement}

Figure 5 shows the terminal voltage analysis results with respect to the number of demagnetized poles at the no-load and constant speed condition. From this result, regular decrease of terminal voltage is confirmed. If the rotor speed is not constant, due to the variation of terminal voltages, accurate demagnetization diagnosis is hard.

\subsection{Proposed demagnetization diagnosis method}

In this study, the proposed diagnosis method uses stator current frequency analysis which caused by the rotating magnetic field of stator winding at the locked rotor condition to detect demagnetization of PM placed in rotor of PMSM as shown in Fig. 6.

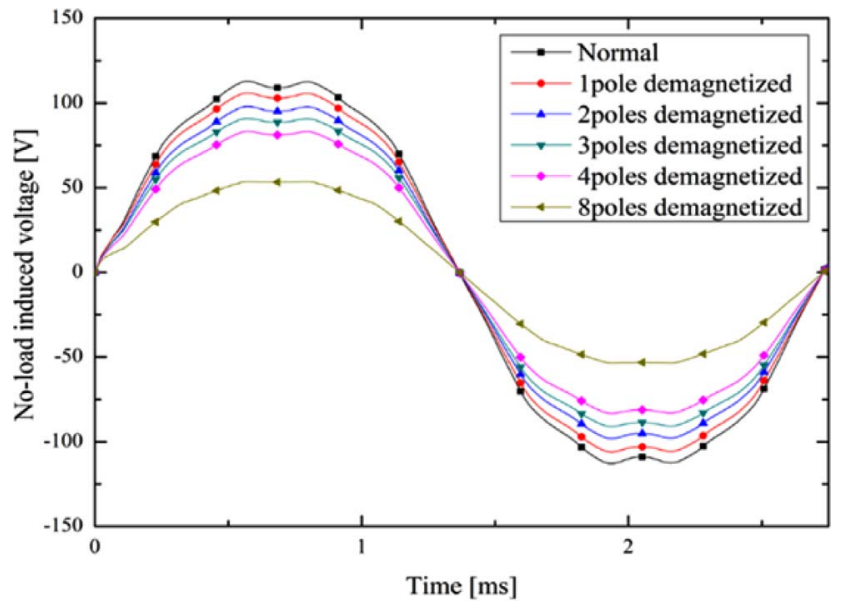

Fig. 5. (Color online) Comparison of no-load induced voltage according to the number of demagnetized poles.

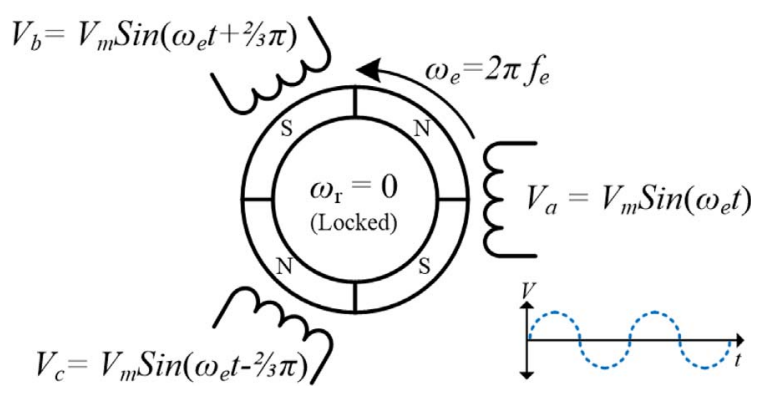

Fig. 6. (Color online) Diagram of proposed diagnosis method.

Where $V_{m}$ is the maximum input voltage, $\omega_{e}$ is angular speed of rotating magnetic field, $f_{e}$ is frequency of input voltage, $\omega_{r}$ is angular speed of rotor respectively.

When the rotating field is applied to PMSM at the locked rotor condition, the input current waveform represents internal flux distribution of PMSM.

Unlike induction motor, starting torque is not produced by rotating magnetic field because the rotating magnetic field is faster than rotor speed. However, permanent magnet can be demagnetized through the negative external magnetic field from stator current.

In order to prevent demagnetization through proposed diagnosis method, the amplitude of input voltage is determined as $80 \%$ lower than rated voltage to reduce the input current of stator winding. In addition, the frequency of input voltage is determined as 3 times higher than synchronous frequency of target PMSM to reduce input current and sampling time for frequency analysis.

\section{Analysis of Simulation Results}

Figure 7 shows the analysis results of input waveform of each model. In Fig. 7, the fundamental frequency of 

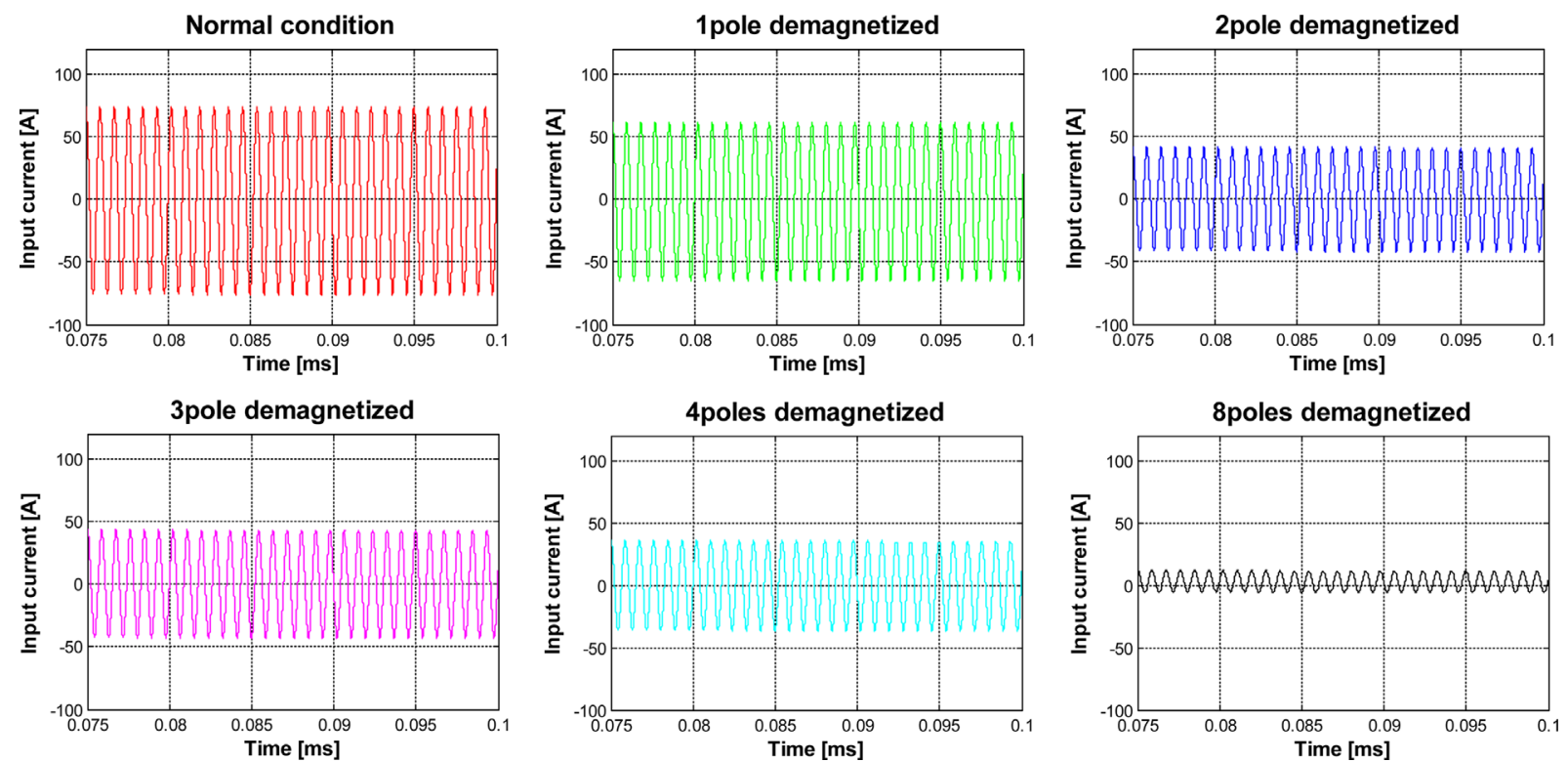

Fig. 7. (Color online) Analysis result of input current waveform.

input voltage is $1.1[\mathrm{kHz}]$.

As the number of demagnetized poles is increased, the irregularly decreased input current is observed. However, the distortion of input current waveform is not detected in time domain.

Figure 8 shows power spectral density of input current waveforms. Power spectral density (PSD) is the variation of a signal's power versus frequency.

The most of demagnetized model result is substantially different from the normal condition result. Demagnetized models represent much higher signal power between the harmonics than normal model. On the other hand, 4poles and 8poles demagnetized model results are similar to normal condition result.

In Fig. 9, the PSD of normal and 1 pole demagnetized model is compared. Harmonics of input current has no considerable difference, but the increase of PSD is shown between each harmonics.

In Fig. 10, the PSD of normal and 8poles demagnetized model is compared. Contrary to 1 pole demagnetized
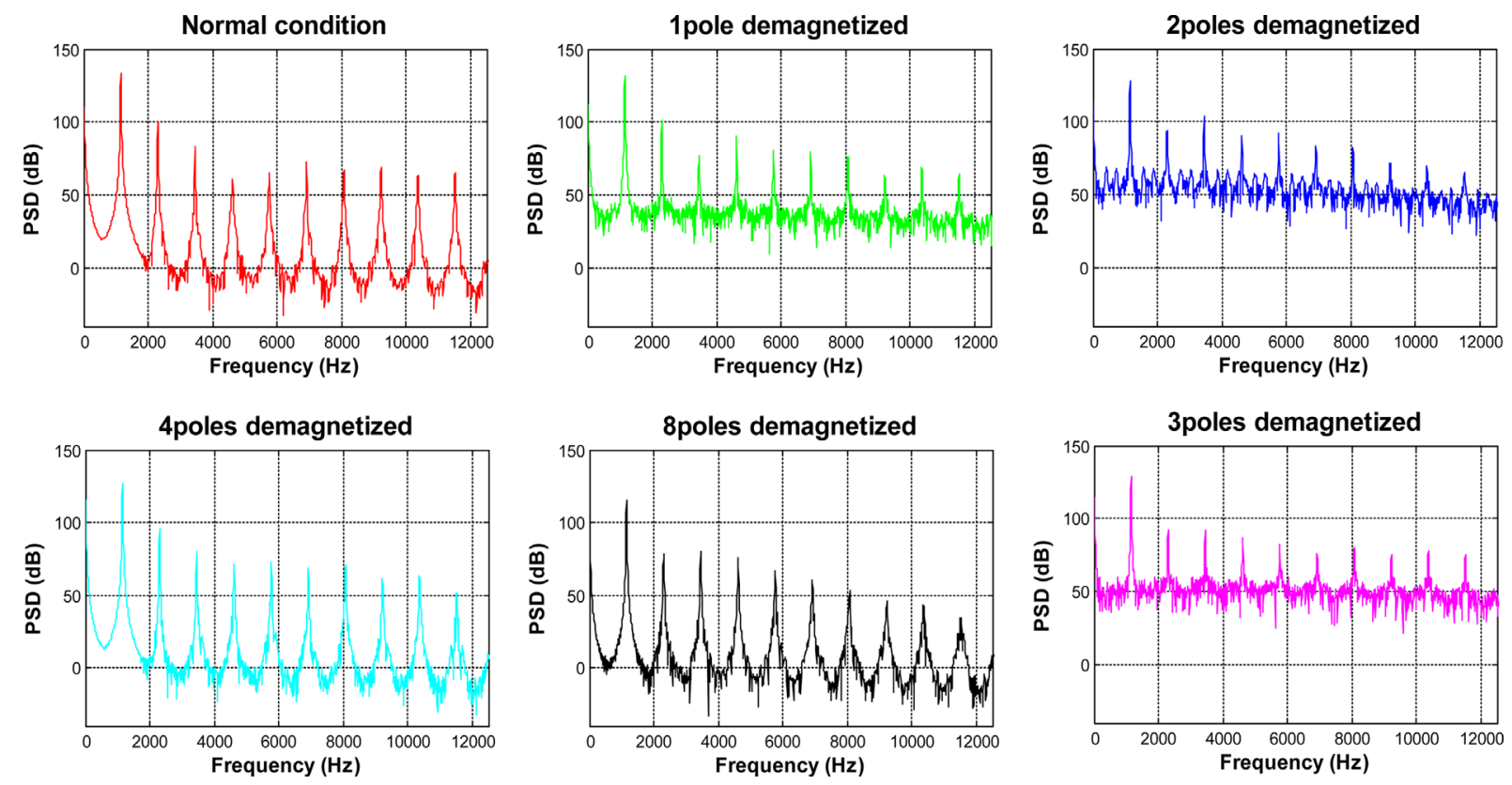

Fig. 8. (Color online) Power spectral density of input current waveform. 


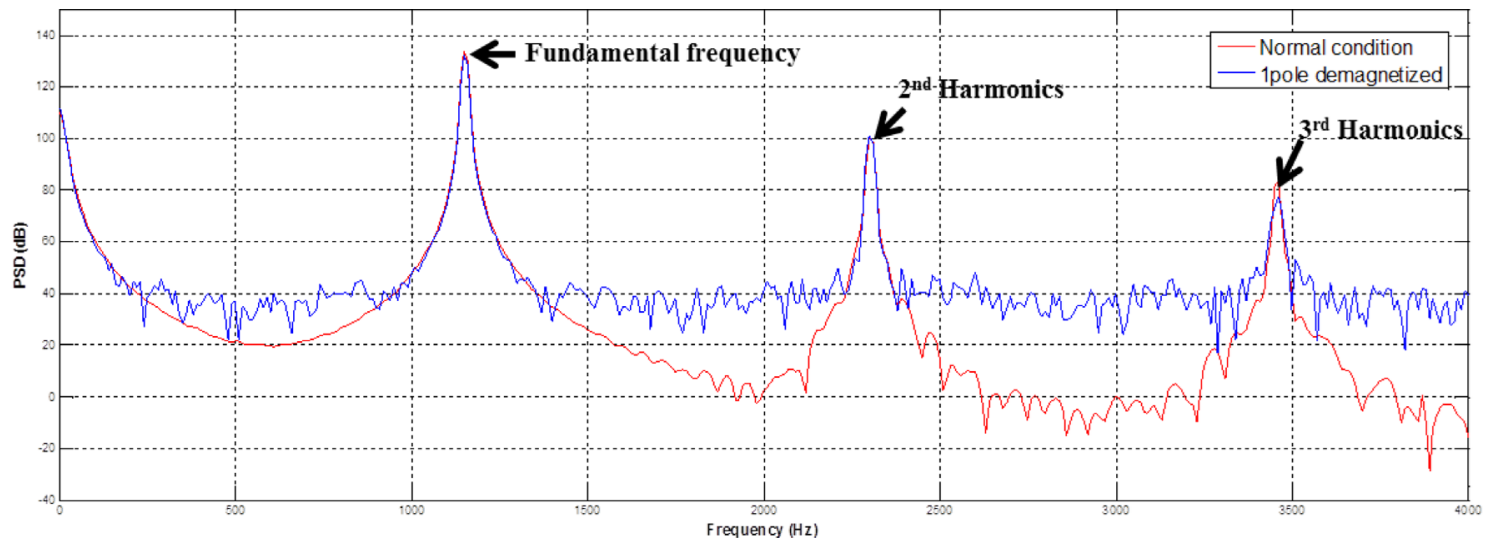

Fig. 9. (Color online) Power spectral density comparison of normal and 1pole demagnetized model.

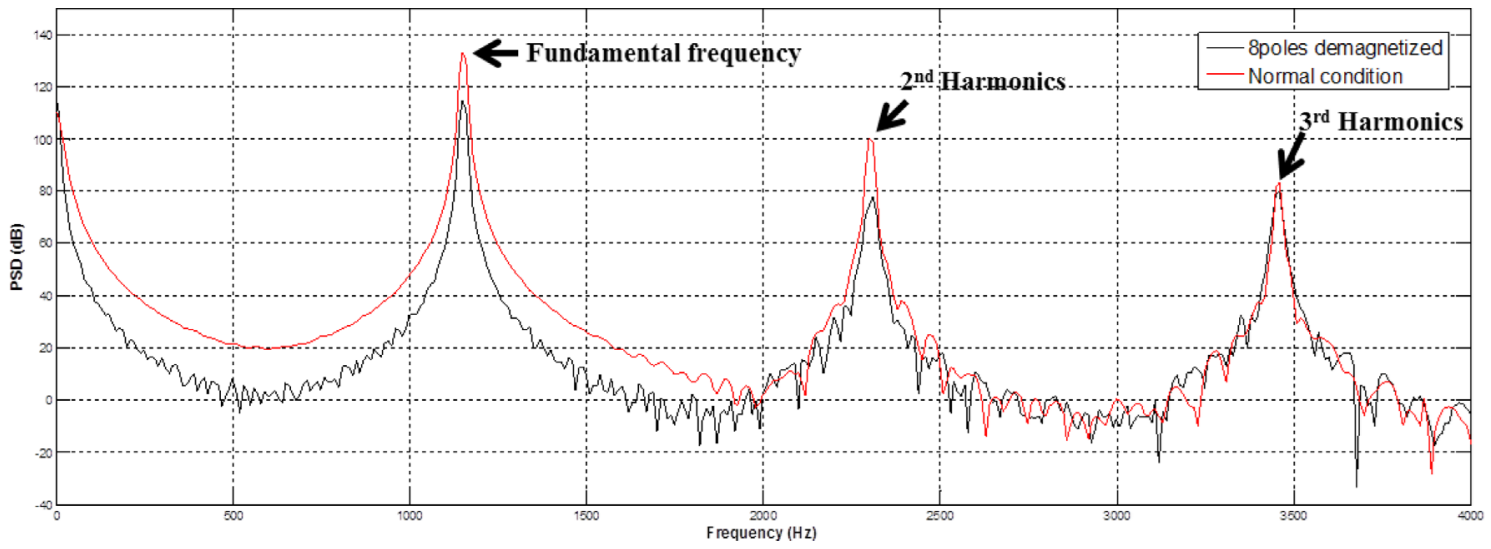

Fig. 10. (Color online) Power spectral density comparison of normal and 8 pole demagnetized model.

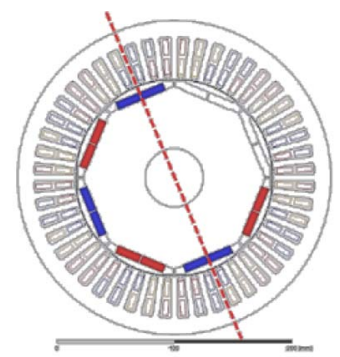

(a)

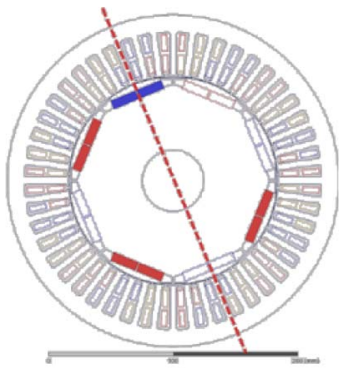

(c)

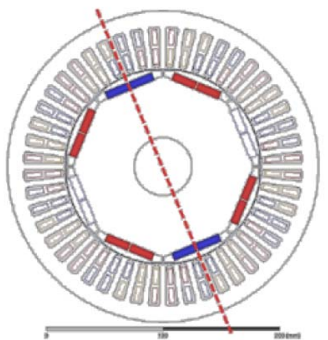

(b)

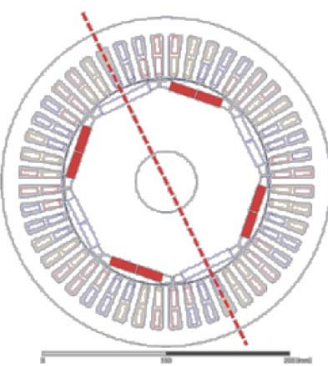

(d)
Fig. 11. (Color online) Comparison of power density according to arrangement of demagnetized poles.
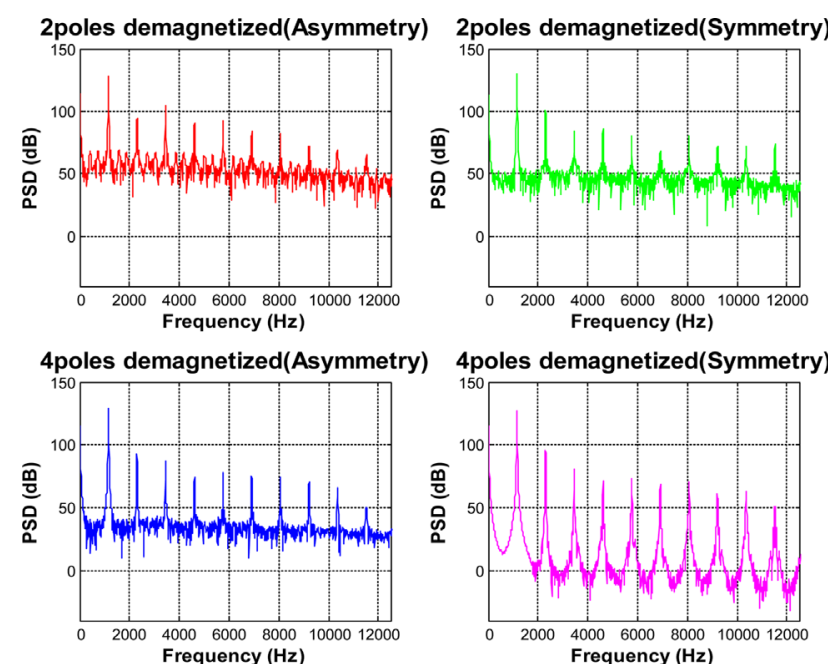

Fig. 12. (Color online) Comparison of power density according to arrangement of demagnetized poles.

model, fundamental frequency and 2nd harmonics of input current has considerable difference.

Figure 11 shows the models to verify the effect of 

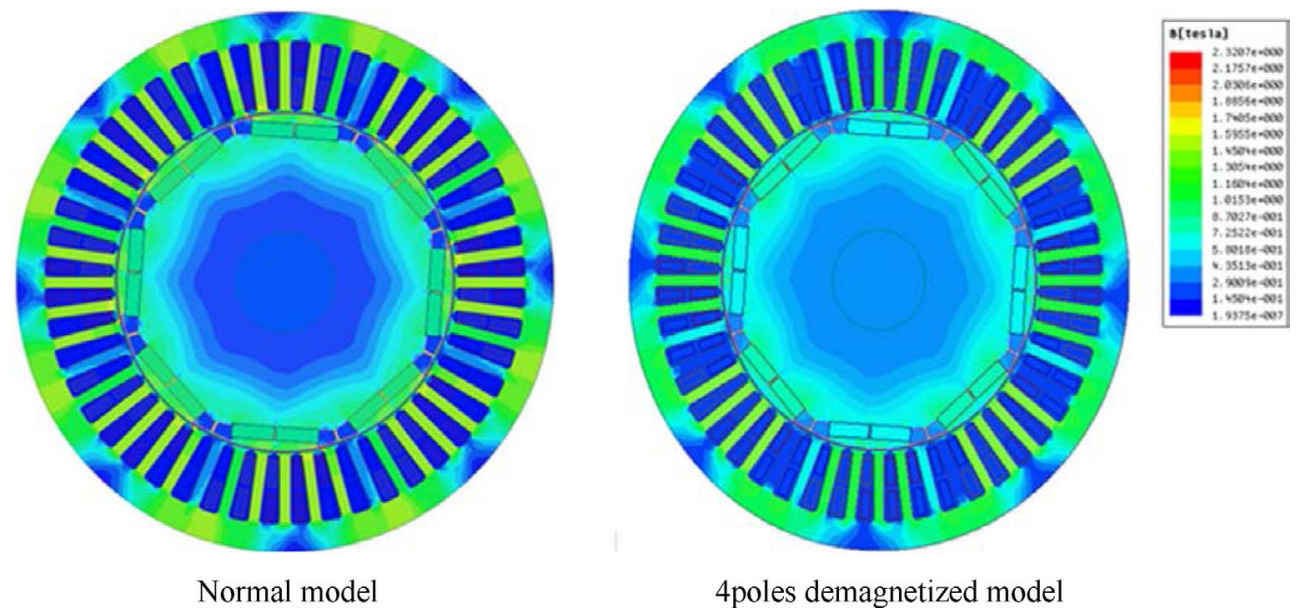

Fig. 13. (Color online) Comparison of flux density distribution.

demagnetized pole arrangement. In Fig. 11, (a) and (c) are asymmetrically demagnetized models. On the other hand, (b) and (d) are symmetrically demagnetized models. The axis of symmetry is indicated by the dotted red line.

In Fig. 12, (a), (b) and (c) are significantly different from the normal condition, but (d) is not. In the case of (d), demagnetized poles are placed at intervals of $90^{\circ}$ in mechanical angle same as one period of electrical angle.

In Fig. 13, flux density distribution analysis results of normal and 4poles demagnetized model are compared. Flux density of normal model is much higher than 4pole demagnetized model, but the distribution of flux density has no considerable difference. Consequently, the PSD of input current between harmonics represents flux density distribution.

\section{Conclusion}

In this study, demagnetization diagnosis method based on the result of locked rotor test and frequency analysis is proposed. Using the proposed method, demagnetized poles can be detected without disassembly of a motor.

In order to verify the proposed demagnetization diagnosis method, each case is distinguished between the normal and fault models by using the FEA.

Most cases are successfully distinguished by PSD comparison between the harmonics. However, 4pole and 8pole demagnetized model requires fundamental frequency and 2nd harmonics comparison.

\section{Acknowledgments}

This research was financially supported by the Ministry of Education (MOE) and National Research Foundation of Korea (NRF) through the Human Resource Training Project for Regional Innovation (NRF-2013H1B8A2028789).

\section{References}

[1] J. R. R. Ruiz, J. Rosero, A. G. Espinosa, and L. Romeral, IEEE Trans. Magn. 45, 7 (2009).

[2] J. Rosero, L. Romeral, J. A. Ortega, and J. C. Urresty, Int. Sym on ISIE (2008).

[3] M. A. S. K. Khan, T. S. Radwan, and M. A. Rahman, Proc. Conf. Rec. IEEE IAS Annual Meeting (2006).

[4] J. Rosero, A. Garcia, L. Romeral, and J. A. Ortega, IEEE WISP'2007 (2007).

[5] Urresty, J. Riba Ruiz, J.-R. Delgado, and M. Romeral L., IEEE Trans. Energy Conversion 27, 1 (2012).

[6] R. Z. Haddad and E. G. Strangas, Diagnostics for Electric Machines, Power Electronics and Drives (SDEMPED) (2013).

[7] B. M. Ebrahimi and J. Faiz, IEEE Trans. Magn. 49, 3 (2013).

[8] K. Yamazaki, T. Fukuoka, K. Akatsu, N. Nakao, and A. Rudeman, IEEE Trans. Magn. 48, 11 (2012).

[9] M. Popescu, M. Hafner, and D. G. Dorrell, IECON 2014 (2014). 